\title{
In Vitro Effects of Calcium Dobesilate on the Responsiveness of Spontaneously Diabetic Rat Aorta
}

\author{
Mercedes Sanz, Emilio Ruiz, Patricia Ganado and Teresa Tejerina* \\ Department of Pharmacology, School of Medicine, Complutense University, 28040 Madrid, Spain \\ Received June 2, 1998 Accepted September 23, 1998
}

\begin{abstract}
We tested the effect of calcium dobesilate (DOBE) in aorta from spontaneously diabetic (BB/wor) rats. The contraction induced by $10^{-6} \mathrm{M}$ noradrenaline (NA) in $\mathrm{BB} /$ wor rats was smaller than that induced in control rats $(1.21 \pm 0.11$ vs $0.82 \pm 0.02 \mathrm{~g}, \mathrm{P}<0.01, \mathrm{n}=8$, respectively) in arteries with intact endothelium. Incubation with DOBE $\left(10^{-4} \mathrm{M}\right)$ impaired the contractions induced by NA in BB/wor rats $(1.21 \pm 0.11$ vs $0.67 \pm 0.01 \mathrm{~g}, \mathrm{P}<0.01, \mathrm{n}=8)$. The effect of DOBE was reversed by $10^{-6} \mathrm{M}$ propranolol $\left(0.67 \pm 0.01\right.$ vs $1.20 \pm 0.60 \mathrm{~g}, \mathrm{P}<0.001, \mathrm{n}=8$, with $10^{-4} \mathrm{M}$ DOBE and $10^{-4} \mathrm{M}$ DOBE plus $10^{-6} \mathrm{M}$ propranolol, respectively). DOBE increased the endothelium-dependent relaxation in arteries from diabetic rats. These findings suggest that DOBE might improve vascular reactivity in $\mathrm{BB} /$ wor rats.
\end{abstract}

Keywords: Spontaneously diabetic rat, Aorta, Calcium dobesilate

It has been suggested that some manifestations of cardiovascular deterioration in diabetes are a consequence of altered sensitivity and/or responsiveness of vascular smooth muscle to neurotransmitters and circulating hormones (1). In this respect, the reactivity of the aorta of the diabetic rat to contracting agents has been extensively studied. MacLeod and McNeill (2) have demonstrated that contractile responses to norepinephrine of the aorta and mesenteric arteries were enhanced in rats with streptozotocin-induced diabetes. In addition, there is controversy about endothelium-dependent relaxation in arteries from diabetic animals. Wakabayashi et al. (3) have reported that endothelium-dependent relaxation of aortas in response to acetylcholine (ACh) was unaffected by the diabetic state. In contrast, Oyama et al. (4) have reported that endothelium-dependent relaxation of diabetic aortas was attenuated with respect to the control.

Previous studies carried out in our group have demonstrated that calcium dobesilate enhances endotheliumdependent relaxation induced by $\mathrm{ACh}$ in isolated rabbit aorta arteries $(5,6)$. The aim of this study was to investigate the in vitro effect of this drug on both contraction and relaxation responses in aortic rings isolated from spontaneously diabetic rats ( $\mathrm{BB} /$ wor rats).

Male genetically diabetic rats (BB/wor rats) from Mölegaard Breeding and Research (Svensved, Denmark)

* To whom correspondence should be addressed. weighing $300.0 \pm 25.0 \mathrm{~g}$ were used. The Bio-Breeding (BB) rat was selected for use in this study because it is a model of spontaneous diabetes that closely resembles type I diabetes in humans. In our study, $50 \%$ of the BB/wor rats developed severe diabetes between days 70 and 120 of the study. Control rats (Wistar) were housed separately and both groups given free access to food and water.

Glucose and ketones in urine were measured each morning; and when the glucose/ketone ratio was $>1$, a blood sample was taken by puncturing of the tail. The sample was tested for glycemia by using Gluco Touch reagent strips (Johnson and Johnson Co., Milpitas, CA, USA). A subcutaneous pellet of insulin (Limplat ${ }^{\circledR}$ Sustained-release Insuline implant, Mölegaard Breeding and Research) was administered when the glycemia level was more than $15 \mathrm{mM}$.

Rats were anesthetized with ethyl ether and killed by cervical dislocation $180 \pm 7$ days after the development of diabetes. The thoracic aorta was isolated. Two types of ring preparation were obtained: the first, those in which the endothelium was preserved $(+\mathrm{E}$ arteries) and the second, those in which the endothelium was removed by rubbing with a glass rod ( $-\mathrm{E}$ arteries) (7). The rings were rapidly placed in Godfraind solution of the following composition: $121 \mathrm{mM} \mathrm{NaCl}, 5.8 \mathrm{mM} \mathrm{KCl}, 14.9 \mathrm{mM}$ $\mathrm{NaHCO}_{3}, 1.22 \mathrm{mM} \mathrm{MgCl} 2,11 \mathrm{mM}$ glucose and $1.25 \mathrm{mM}$ $\mathrm{CaCl}_{2}$. Adherent fat and surrounding tissue were cleaned off, and the rings were then suspended between two 
stainless steel hooks in organ baths containing $10 \mathrm{ml}$ of Godfraind solution. The solution was kept at $36 \pm 0.5^{\circ} \mathrm{C}$ and gassed continuously with a $95 \% \mathrm{O}_{2}-5 \% \mathrm{CO}_{2}$ gas mixture. The aorta rings were mounted under $1-\mathrm{g}$ tension. Each preparation was allowed to equilibrate for $60-90$ min. Contractile responses were measured isometrically by means of force-displacement transducers (model FT 03; Grass Instrument Co., Quincy, MA, USA) and recorded on a Grass polygraph as previously described (8). The isometric force was also digitalized by a MacLab A/D converter (Chart v3.2; A.D. Instruments Pty., Ltd., Castle Hill, Australia) and stored and displayed on a Mackintosh computer (9).

We tested the effect of diabetes on a single submaximal contraction induced with $10^{-6} \mathrm{M}$ noradrenaline (NA) in both groups of arteries ( $+E$ and $-E$ ). After being washed out, the rings were incubated with calcium dobesilate (DOBE) $\left(10^{-5}\right.$ or $\left.10^{-4} \mathrm{M}\right)$ for $45 \mathrm{~min}$, and then the contraction with NA was repeated.

In view of the results obtained, we studied the implication of the L-Arg-NO pathway and $\beta$-adrenergic receptors in the effect of DOBE. For this, we repeated the contraction induced with $10^{-6} \mathrm{M} \mathrm{NA}$ in the presence of DOBE $\left(10^{-4} \mathrm{M}\right.$ ) plus $10^{-4} \mathrm{M} N$-nitro-L-arginine methyl ester (LNAME) (NO-synthase inhibitor) or plus $10^{-6} \mathrm{M}$ propranolol ( $\beta$-blocker).

In order to investigate whether diabetes altered both endothelium-dependent and endothelium-independent relaxant responses and if this were the case, whether or not incubation with different concentrations of DOBE could prevent these alterations, an initial contraction by $10^{-6} \mathrm{M}$ NA was induced. When the plateau was reached, the following cumulative relaxation curves were made by adding aliquots of the relaxing agonist: i) Concentrationresponse curves to $\mathrm{ACh}\left(10^{-8}-10^{-4} \mathrm{M}\right)$ and ii) Concentration-response curves to sodium nitroprusside (SNP) $\left(10^{-8}-10^{-4} \mathrm{M}\right)$.

After being washed out, the rings were incubated with DOBE at $10^{-5}$ or $10^{-4} \mathrm{M}$ for $45 \mathrm{~min}$ and the procedure repeated.

The following drugs were used: calcium dobesilate was a generous gift from Laboratorios Esteve (Barcelona, Spain); and noradrenaline bitartrate, SNP, propranolol, acetylcholine chloride and L-NAME were all from Sigma (St. Louis, MO, USA). The drugs were dissolved in deionized water; working solutions were made in Godfraind solution. Ascorbic acid $\left(10^{-4} \mathrm{M}\right)$ was added to each daily prepared solution of NA.

The results are expressed throughout as the mean \pm S.E.M. of 7-9 rats in each group. All protocols concerning animals were approved by the Complutense University of Madrid (EEC official registration 28079$15 \mathrm{ABC}$ ). Comparisons between the different groups were performed by Student's $t$-test for unpaired data or two way ANOVA when needed. Differences were considered significant when $P<0.05$.

As shown in Fig. 1A, in $+\mathrm{E}$ arteries, the contraction induced by NA increased in $\mathrm{BB} /$ wor rats with respect to that in control rats $(1.21 \pm 0.11$ vs $0.82 \pm 0.02 \mathrm{~g}, \mathrm{P}<0.01$, $n=8$, respectively). In $+E$ rings obtained from $B B /$ rats incubated with $10^{-5} \mathrm{M}$ DOBE, the contraction was $0.96 \pm 0.10 \mathrm{~g}$ and with $10^{-4} \mathrm{M}$ DOBE, this contraction was $0.67 \pm 0.01 \mathrm{~g}(\mathrm{P}<0.01, \mathrm{n}=8)$. However, in $-\mathrm{E}$ arteries, the contraction induced by NA decreased slightly, but not significantly, in rings obtained from $\mathrm{BB} /$ wor rats with respect to that in control rats $(1.01 \pm 0.10 \mathrm{vs}$
A



B

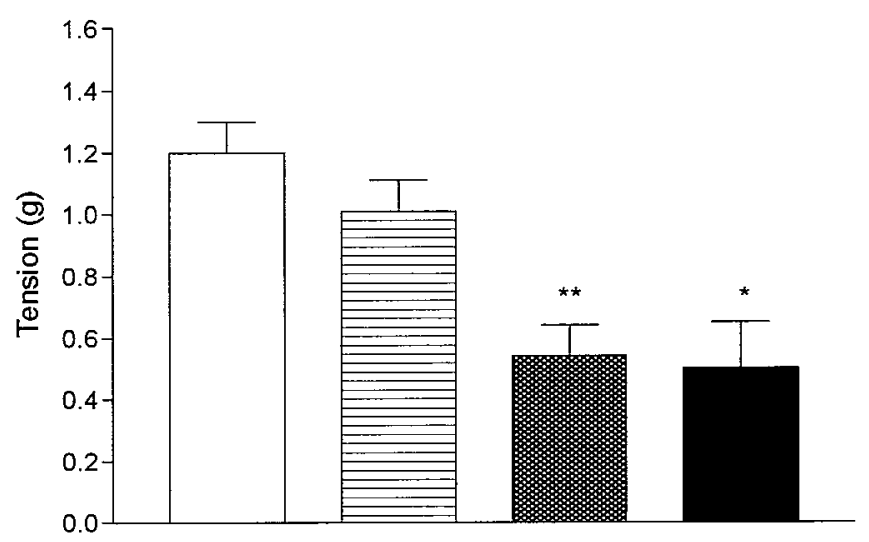

Fig. 1. Effect of DOBE $\left(10^{-5}\right.$ or $\left.10^{-4} \mathrm{M}\right)$ on contractions induced by $10^{-6} \mathrm{M}$ NA in both groups of aortic rings, +E (panel A) and $-\mathrm{E}$ (panel $\mathrm{B}$ ) obtained from diabetic rats (BB/wor rats). Each bar shows the mean \pm S.E.M. of $7-9$ experiments. ${ }^{*} \mathrm{P}<0.05$,

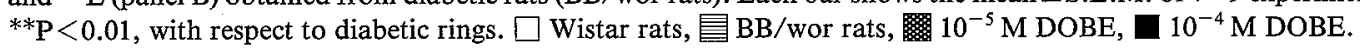




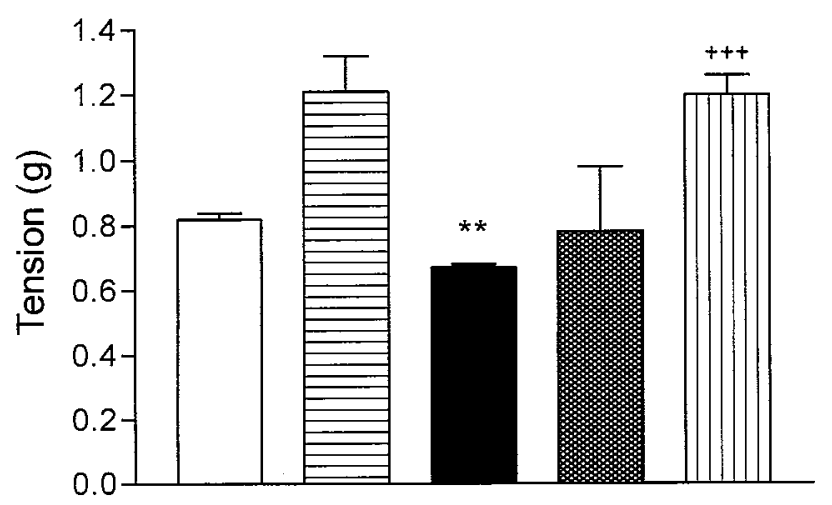

Fig. 2. Effect of L-NAME $\left(10^{-4} \mathrm{M}\right)$ or propranolol $\left(10^{-6} \mathrm{M}\right)$ on the preventive effect of DOBE on contractions induced by $10^{-6} \mathrm{M}$ $\mathrm{NA}$ in aortic rings obtained from diabetic rats (BB/wor rats). Each bar shows the mean \pm S.E.M. of $7-9$ experiments. ${ }^{* *} \mathrm{P}<0.01$, with respect to diabetic rings, ${ }^{+++} \mathrm{P}<0.001$, with respect to NA-induced contraction in the presence of DOBE. $\square$ Wistar rats, $\mathrm{EB}$ /wor rats, $10^{-4} \mathrm{M}$ DOBE, $\mathrm{DOBE}+10^{-4} \mathrm{M} \mathrm{L}$-NAME, 䙹 DOBE + $10^{-6} \mathrm{M}$ propranolol.

$1.20 \pm 0.10 \mathrm{~g}$ in rings from $\mathrm{BB} /$ wor and control rats, respectively). In addition, when aortic rings from $\mathrm{BB} /$ wor rats were incubated in the presence of DOBE, the contraction induced by NA decreased from $1.01 \pm 0.10$ to $0.54 \pm 0.10 \mathrm{~g}(\mathrm{P}<0.01, \mathrm{n}=8)$ and $0.50 \pm 0.15 \mathrm{~g}(\mathrm{P}<0.05$, $\mathrm{n}=8$ ) for $10^{-5} \mathrm{M}$ DOBE and $10^{-4} \mathrm{M}$ DOBE, respectively (Fig. 1B).

In order to rule out any action on the endothelium, we tried to reverse the effect of DOBE in the presence of
L-NAME (NO synthesis inhibitor). Figure 2 shows that L-NAME did not significantly reverse the effect of DOBE on the contraction induced by NA $(0.67 \pm 0.01$ vs $0.76 \pm 0.20 \mathrm{~g}$ in $10^{-4} \mathrm{M}$ DOBE and DOBE plus LNAME-treated arteries, respectively).

In addition, in arteries incubated with DOBE $\left(10^{-4}\right.$ $\mathrm{M})$ and with $\mathrm{DOBE}\left(10^{-4} \mathrm{M}\right)$ plus $10^{-6} \mathrm{M}$ propranolol, the contractions induced by $10^{-6} \mathrm{M}$ NA were $0.67 \pm 0.01$ and $1.20 \pm 0.60 \mathrm{~g}(\mathrm{P}<0.001, \mathrm{n}=8)$, respectively.

In arteries obtained from control rats, ACh $\left(10^{-8}-\right.$ $10^{-4} \mathrm{M}$ ) caused a concentration-dependent relaxation; the maximal relaxation of $78.0 \pm 9.0 \%$ being reached with $10^{-4} \mathrm{M}$ ACh. In arteries obtained from $\mathrm{BB} /$ wor rats, the concentration-response curve was significantly $[\mathrm{F}(1,15$ $=5.3), \mathrm{P}<0.05$ ] shifted upwards and to the left, the maximal relaxation being $50.0 \pm 11.0 \%$. In these arteries, after incubation with DOBE $\left(10^{-5}\right.$ or $\left.10^{-4} \mathrm{M}\right)$, the concentration-response curve induced by $\mathrm{ACh}$ was significantly $[F(1,14)=5.3, P<0.05$ and $F(1,10)=10.1$, $\mathrm{P}<0.05$ with DOBE at $10^{-5}$ and $10^{-4} \mathrm{M}$, respectively] shifted downwards and to the right, compared with the curve of control diabetic rats (Fig. 3A).

On the other hand, despite the maximal effects being similar between the groups, the sensitivity to SNP decreased in diabetic rings and in diabetic rings incubated with DOBE, compared with control nondiabetic rings. Thus the $\mathrm{EC}_{50}$ values were $5.1 \pm 0.2 \times 10^{-8}$ vs $4.9 \pm 0.1 \times$ $10^{-7} \mathrm{M}(\mathrm{P}<0.05, \mathrm{n}=8)$ in control nondiabetic rings and diabetic rings, respectively. In arteries isolated from diabetic rats that were incubated with $\mathrm{DOBE}$, the $\mathrm{EC}_{50}$
A

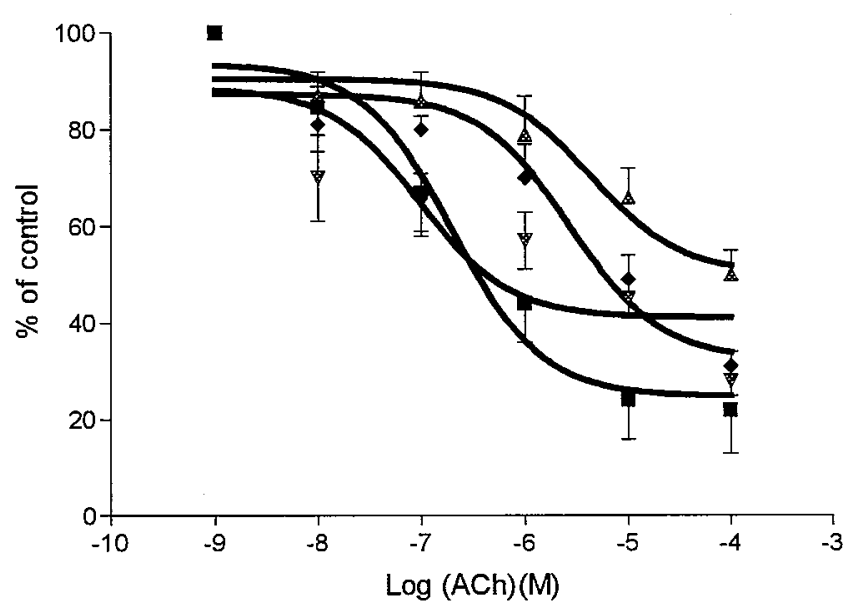

B

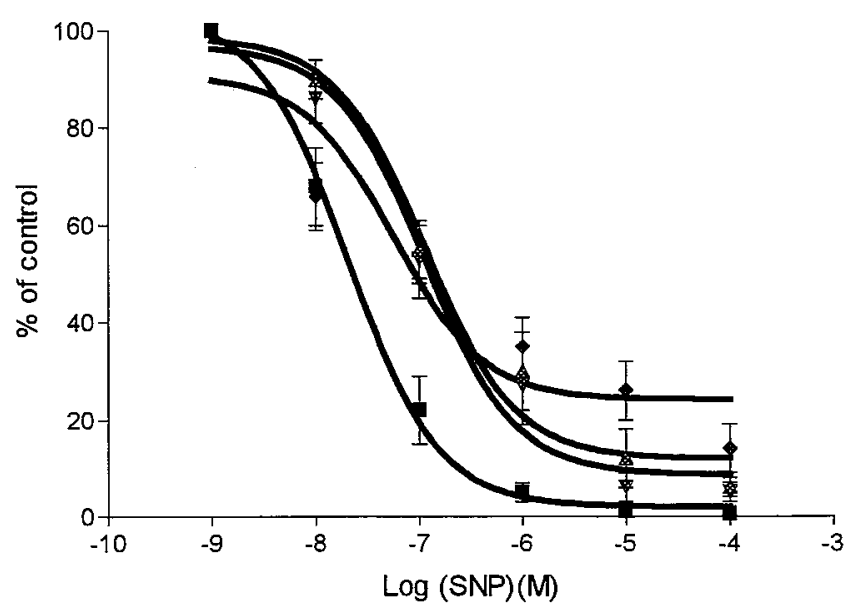

Fig. 3. Effect of DOBE $\left(10^{-5}\right.$ or $\left.10^{-4} \mathrm{M}\right)$ on endothelium-dependent relaxation induced by $\mathrm{ACh}\left(10^{-8}-10^{-4} \mathrm{M}\right)(\mathrm{panel} \mathrm{A})$ or endothelium-independent relaxation induced by SNP $\left(10^{-8}-10^{-4} \mathrm{M}\right)$ (panel B) in aortic rings obtained from diabetic rats (BB/wor rats). Each point shows the mean \pm S.E.M. of $7-9$ experiments. $\square$ wistar rats, $\Delta \mathrm{BB} /$ wor rats, $\nabla 10^{-5} \mathrm{M} \mathrm{DOBE}$, $10^{-4} \mathrm{M}$ DOBE. 
values were $4.8 \pm 0.2 \times 10^{-7} \mathrm{M}(\mathrm{P}<0.05, \mathrm{n}=8$, compared with control nondiabetic rings) and $5.2 \pm 0.1 \times 10^{-7} \mathrm{M}$ ( $P<0.05, n=8$ compared with control nondiabetic rings) for DOBE at $10^{-5}$ and $10^{-4} \mathrm{M}$, respectively (Fig. 3B).

Aorta from streptozotocin-induced diabetic rats have been found to show an attenuated maximal contractile response to NA (10), no changes (11) and even an increase in response to NA (12). The results of this work show that NA-induced contractions are increased in aortic rings isolated from diabetic rats with respect to control (nondiabetic) rats only in arteries with intact endothelium. This fact suggests that NA may induce the release of a contracting factor from endothelia which could, in turn, increase the noradrenaline-induced contraction.

Our results concerning the increased contraction induced by NA are in disagreement with those of other authors who did not find any difference between aortic rings from control and $\mathrm{BB}$ rats $(13,14)$. A possible explanation might be that those previous studies were carried out for 60-90 days, while in our study, the rats were maintained in a diabetic state for $180 \pm 7$ days. In this way, the results of some studies have suggested that the influence of diabetes on vascular reactivity varies with the duration of the diabetic state (2).

On the other hand, when diabetic aortic rings were incubated in the presence of DOBE, we found a decrease in the contractile responses induced by NA only with the highest concentration of DOBE $\left(10^{-4} \mathrm{M}\right)$ in arteries with intact endothelium and with both concentrations in arteries without endothelium, showing that DOBE exerts its effect on contraction by acting on vascular smooth muscle cells. We tried to demonstrate the hypothesis that DOBE could act by modulating $\beta$-adrenergic receptors in vascular smooth muscle. Propranolol completely reversed the effect of DOBE on NA-induced contraction, demonstrating that DOBE acts on $\beta$-adrenergic receptors.

On the other hand, the majority of studies using $\mathrm{BB} /$ wor rats have shown a specific impairment of endothelium-dependent relaxation in conduit arteries such as the aorta $(13,14)$. In this work, the relaxation induced by $\mathrm{ACh}$ was significantly attenuated in diabetic rings compared with nondiabetic control rings and incubation with DOBE significantly enhanced this relaxation, suggesting that DOBE acts on NO synthesis, as we previously reported was the case in isolated rabbit aorta arteries (5, 6). Furthermore, the effect of DOBE on endothelium-independent relaxation was also tested and an impairment in the sensitivity to SNP found in diabetic rings (with or without the presence of DOBE) compared with control nondiabetic rings. These results suggest that not only is the L-Arg-NO pathway altered in the diabetic state after $180 \pm 7$ days but also the NO-cGMP pathway.

Finally, in conclusion, in spontaneously diabetic rat aorta, calcium dobesilate decreases the contractile responses induced by NA through a mechanism that involves $\beta$-adrenergic receptors. On the other hand, the incubation with DOBE improves the endothelium-dependent relaxation in diabetic rat aortic rings.

\section{Acknowledgments}

This work was supported in part by a FISS grant $(96 / 2047)$ and by a grant from Laboratorios Esteve.

\section{REFERENCES}

1 Weidman P, Piccoli C, Keuschm M, Mutagic M, Grimm M, Meier A and Zeigler W: Sodium-volume factor, cardiovascular reactivity and hypotensive mechanism of diuretic therapy in mild hypertension associated with diabetes. Am J Med 67, 779-783 (1979)

2 MacLeod $\mathrm{K}$ and McNeill M: The influence of chronic experimental diabetes on contractile responses of rat isolated blood vessels. Can J Physiol Pharmacol 63, 52-55 (1985)

3 Wakabayashi I, Hatake K, Kimura N, Kakishita E and Nagai $\mathrm{K}$ : Modulation of the vascular tonus by the endothelium in experimental diabetes. Life Sci 40, 603-610 (1987)

4 Oyama Y, Kawasaki H, Hattori Y and Kanno M: Attenuation of endothelium-dependent relaxation in aorta from diabetic rats. Eur J Pharmacol 131, 75-82 (1986)

5 Ruiz E, Lorente $\mathrm{R}$ and Tejerina T: Effects of calcium dobesilate on the synthesis of endothelium-dependent relaxing factors in rabbit isolated aorta. Br J Pharmacol 121, $711-716$ (1997)

6 Ruiz $\mathrm{E}$ and Tejerina T: Calcium dobesilate increases endothelium-dependent relaxation in isolated rabbit aorta. Gen Pharmacol 30, 713-718 (1997)

7 Furchgott R, Cherry P, Zawadki J and Jothianandan D: Endothelial cells as mediators of vasodilatation of arteries. J Cardiovasc Pharmacol 6, S336-S341 (1984)

8 Tejerina T, Sesin J, Delgado C and Tamargo J: Effect of milrinone contractility and ${ }^{45} \mathrm{Ca}^{2+}$ movements in the isolated rabbit aorta. Eur J Pharmacol 148, 239-245 (1988)

9 Ruiz $\mathrm{E}$ and Tejerina T: Possible role of $l$-citrulline in rabbit vascular smooth muscle. Br J Pharmacol 125, 186-192 (1998)

10 Ramanadhan S, Lyness W and Tenner T: Alterations in aortic and tail artery reactivity to agonists after streptozotocin treatment. Can J Physiol Pharmacol 62, $418-423$ (1984)

11 Abebe W and Mcleod K: Protein kinase C-mediated contractile response of arteries from diabetic rats. $\mathrm{Br} \mathrm{J}$ Pharmacol 101, 465-471 (1987)

12 Harris $\mathrm{K}$ and MacLedo K: Influence of the endothelium on contractile responses of arteries from diabetic rats. Eur J Pharmacol 153, 55-64 (1988)

13 Koltai M, Rosen P, Hadhazy P, Ballagi-Pordany G, Koszeghy $A$ and Pogatsa G: Relationship between vascular adrenergic receptors and prostaglandin biosyntheses in canine diabetic coronary arteries. Diabetologia 31, 681-686 (1988)

14 Durante W, Sen A and Sunahara F: Impairment of endothelium-dependent relaxation in aortae from spontaneously diabetic rats. Br J Pharmacol 94, 463-468 (1988) 